\title{
MULTILINEAR EXTENSIONS OF GROTHENDIECK'S THEOREM
}

\author{
FERNANDO BOMBAL, DAVID PÉREZ-GARCÍA, AND IGNACIO VILLANUEVA
}

\begin{abstract}
We introduce a new class of multilinear $p$-summing operators, which we call multiple p-summing. Using them, we can prove several multilinear generalizations of Grothendieck's "fundamental theorem of the metric theory of tensor products". Several applications and improvements of previous results are given.
\end{abstract}

\section{INTRODUCTION AND NOTATION}

In [7], Grothendieck stated what he called "the fundamental theorem of the metric theory of tensor products", to be known later as Grothendieck's Theorem or Grothendieck's Inequality. In [8], Lindenstrauss and Pełcyński gave a detailed proof of this result and stated some of its consequences and equivalent formulations, making use of the theory $\mathcal{L}_{p}$-spaces and using also the $p$-summing operators recently introduced in [14]. Since then, several equivalent formulations and different proofs have been obtained. [5] and [16] provide excellent expositions on this and related topics.

Grothendieck's Theorem can be seen as a matrix inequality associated to certain bilinear operators. In the seventies, several authors investigated multilinear extensions of Grothendieck's matrix Inequality (see, for instance, [2], [3], [19] and the references therein).

Later on, motivated by the growth of the multilinear and polynomical theory of Banach spaces, different authors started the study of the $p$-summing multilinear operators between Banach spaces (see [1], [6], [10], [15], [17]). For some of these $p$ summing multilinear operators a factorization result extending Pietsch DominationFactorization Theorem [5, Theorems 2.12 and 2.13] holds, but no multilinear version of Grothendieck's Theorem in terms of these operators is known, except for a special case presented in [2] (see also [11]).

In this note we introduce a new definition of $p$-summing multilinear operators (although the origin of this definition can be found in [17, Definition 3.6]), which we call multiple p-summing operators. In this paper we focus on Grothendieck type theorems related to this class. As this kind of theorems shows that the class is, in some sense, big enough, their interest stems from the fact that this class is actually quite small. For example, the Aron-Berner extension of any of these operators remains in the image space and, on $\mathcal{L}_{\infty}$ spaces, multiple 1 -summing operators are exactly the integral operators (see [20]). For these and other results associated to

1991 Mathematics Subject Classification. 47H60, 46B25, 46B07.

Key words and phrases. Grothendieck's inequality, $p$-summing operators, absolutely summing operators, multilinear operators, $\mathcal{L}_{p}$ spaces.

All three authors were partially supported by DGICYT grant BMF2001-1284. 
this class of multilinear operators, we refer the reader to [12] and [13]. Thanks to this behavior, our feeling is that, for many applications, this might be the "right" generalization of the definition of $p$-summing operator.

The paper is organized as follows. In Section 2 we define multiple $p$-summing multilinear operators and prove the basic properties we later need. In Section 3 we prove that every multilinear operator from the product of $\mathcal{L}_{\infty}$-spaces to a cotype 2 space is multiple 2 -summing. With the techniques developed, we obtain great improvements of $[4$, Theorem 6] and [4, Corollary 7]. In Section 4 we generalize Krivine's version of Grothendieck's inequality. As an application we give a simpler proof of one of the multilinear Grothendieck's inequalities stated in [19]. Our proof works for both the real and the complex case and it shows that the constant involved, $K_{G}^{n}$, is optimal, improving therefore the previous proofs (Tonge's proof works only in the complex case and gives a worse constant and Carne's proof, although it gives the same constant, is not as simple and does not allow to obtain easily the optimality of the constant). In Section 5 we show that every multilinear operator from the product of $\mathcal{L}_{1}$-spaces to a Hilbert space is multiple 2-summing, which can be regarded as an extension of the "little Grothendieck's theorem" [16, Theorem 5.10]. Using that and a composition theorem, we show that every such multilinear operator is actually multiple 1-summing, which extends another equivalent form of Grothendieck's Theorem ([16, Theorem 5.12]). As an application of this we state another multilinear generalization of Grothendieck's matrix inequality which seems to be new.

The notations and terminology used along the paper will be the standard in Banach space theory, as for instance in [5], which is our main source for unexplained notation. This book is also our main reference for basic facts and definitions concerning most of the topics in this paper. However, before going any further, we shall establish some terminology: $X_{i}, Y$ will always be Banach spaces. As usual, $X_{1} \otimes_{\pi} \cdots \otimes_{\pi} X_{k}$ stands for the projective tensor product of the Banach spaces $X_{1}, \ldots, X_{k}$. Continuous $k$-linear mappings between Banach spaces will be called $k$-linear operators. Given a Banach space $X, X^{*}$ stands for its topological dual and $B(X)$ or $B_{X}$ denotes its unit ball.

Given $X$ and $1 \leq p \leq \infty$, we say that a sequence $\left(x_{n}\right)_{n} \subset X$ is strongly $p$ summable if $\left(\left\|x_{n}\right\|\right)_{n} \in \ell_{p}$. We denote by $\ell_{p}(X)$ the Banach space of all such sequences endowed with the norm

$$
\left\|\left(x_{n}\right)_{n}\right\|_{p}=\left(\sum_{n}\left\|x_{n}\right\|^{p}\right)^{\frac{1}{p}} .
$$

We say that $\left(x_{n}\right)_{n}$ is weakly $p$-summable if, for every $x^{*} \in X^{*},\left(\left\langle x^{*}, x_{n}\right\rangle\right)_{n} \in \ell_{p}$. We call $\ell_{p}^{\omega}(X)$ the Banach space of all such sequences endowed with the norm

$$
\left\|\left(x_{n}\right)_{n}\right\|_{p}^{\omega}=\sup \left\{\left(\sum_{n}\left\langle x^{*}, x_{n}\right\rangle^{p}\right)^{\frac{1}{p}}: x^{*} \in B_{X^{*}}\right\} .
$$

Given $1 \leq p \leq q \leq \infty$, we write $\Pi_{p}(X, Y)$ for the Banach space of the $p$ summing operators from $X$ into $Y$, and $\Pi_{(q, p)}(X, Y)$ for the Banach space of the $(q, p)$-summing operators. Given $T \in \Pi_{p}(X, Y), \pi_{p}(T)$ denotes its $p$-summing norm. 
We will note by $K_{G, \mathbb{R}}$ and $K_{G, \mathbb{C}}$ the least constants for which, in the real and in the complex case respectively, Grothendieck's inequality is valid as stated for instance in $[5,1.14]$. We will simply use $K_{G}$ when referring to both cases.

Let $1 \leq p \leq \infty$ and $\lambda>1$. A Banach space $X$ is said to be an $\mathcal{L}_{p, \lambda}$ space if every finite dimensional subspace $E \subset X$ is contained in a finite dimensional space $F \subset X$ for which there exists an isomorphism $v: F \longrightarrow \ell_{p}^{\operatorname{dim} F}$ such that $\|v\|\left\|v^{-1}\right\|<\lambda$. We say that $X$ is an $\mathcal{L}_{p}$ space if it is an $\mathcal{L}_{p, \lambda}$ space for some $\lambda>1$. Clearly, $L_{p}(\mu)$ is the basic example of an $\mathcal{L}_{p}$-space.

Given $n, m_{1}, \ldots, m_{n} \in \mathbb{N},\left(x_{i_{1}, \ldots, i_{n}}\right)_{i_{1}, \ldots, i_{n}=1}^{m_{1}, \ldots, m_{n}}$ denotes a multiindex sequence with the index $i_{j}$ varying from 1 to $m_{j}(1 \leq j \leq n)$. $\sum_{i_{1}, \ldots, i_{n}=1}^{m_{1}, \ldots, m_{n}} x_{i_{1}, \ldots, i_{n}}$ means the same as $\sum_{i_{1}=1}^{m_{1}} \cdots \sum_{i_{n}=1}^{m_{n}} x_{i_{1}, \ldots, i_{n}}$. Further notation will be introduced when needed.

\section{Definitions and Basic Facts}

We start with our definition.

Definition 2.1. Let $1 \leq q_{1}, \ldots, q_{n} \leq p<+\infty$. A multilinear operator $T: X_{1} \times$ $\cdots \times X_{n} \longrightarrow Y$ is multiple $\left(p ; q_{1}, \ldots, q_{n}\right)$-summing if there exists a constant $K>0$ such that, for every choice of sequences $\left(x_{i_{j}}^{j}\right)_{i_{j}=1}^{m_{j}} \subset X_{j}$ the following relation holds

$$
\left(\sum_{i_{1}, \ldots, i_{n}=1}^{m_{1}, \ldots, m_{n}}\left\|T\left(x_{i_{1}}^{1}, \ldots, x_{i_{n}}^{n}\right)\right\|^{p}\right)^{\frac{1}{p}} \leq K \prod_{j=1}^{n}\left\|\left(x_{i_{j}}^{j}\right)_{i_{j}=1}^{m_{j}}\right\|_{q_{j}}^{\omega}
$$

In that case, we define the multiple $\left(p ; q_{1}, \ldots, q_{n}\right)$-summing norm of $T$ by

$$
\pi_{\left(p ; q_{1}, \ldots, q_{n}\right)}(T)=\min \{K: K \text { verifies }(1)\}
$$

Some comments are in order:

If $q_{j}>p$, only the zero operator can satisfy (1). This is the reason to introduce the hypothesis $1 \leq q_{1}, \ldots, q_{n} \leq p<+\infty$.

A multiple $(p ; q, \ldots, q)$-summing operator will be called multiple $(p, q)$-summing and we write $\pi_{(p, q)}$ for the associated norm. Moreover, a multiple $(p, p)$-summing operator will be called multiple p-summing and we write $\pi_{p}$ for the associated norm. We have that the class $\Pi_{\left(p ; q_{1}, \ldots, q_{n}\right)}^{n}\left(X_{1}, \ldots, X_{n} ; Y\right)$ of multiple $\left(p ; q_{1}, \ldots, q_{n}\right)$ summing $n$-linear operators is a Banach space with its associated norm $\pi_{\left(p ; q_{1} \ldots, q_{n}\right)}$.

Just as in [11, Prop. 3.2, 3.4] we obtain the following proposition, which allows us to consider either finite or infinite sequences in the definition of our operators.

Proposition 2.2. Let $1 \leq q_{1}, \ldots, q_{n} \leq p<+\infty$ and consider a multilinear operator $T: X_{1} \times \cdots \times X_{n} \longrightarrow Y$. The following are equivalent.

i) $T$ is $\left(p ; q_{1}, \ldots, q_{n}\right)$-summing.

ii) For every choice of sequences $\left(x_{i_{j}}^{j}\right)_{i_{j}=1}^{\infty} \in \ell_{q_{j}}^{\omega}\left(X_{j}\right)$, we have that

$$
\left(T\left(x_{i_{1}}^{1}, \ldots, x_{i_{n}}^{n}\right)\right)_{i_{1}, \ldots, i_{n}=1}^{\infty} \in \ell_{p}\left(\mathbb{N} \times{ }_{n}, \times \mathbb{N} ; Y\right)
$$

In that case, we obtain that the induced multilinear mapping

$$
\hat{T}: \ell_{q_{1}}^{\omega}\left(X_{1}\right) \times \cdots \times \ell_{q_{n}}^{\omega}\left(X_{n}\right) \longrightarrow \ell_{p}(\mathbb{N} \times \underset{n}{\ldots}, \times \mathbb{N} ; Y)
$$


given by $\hat{T}\left(\left(x_{i_{1}}^{1}\right)_{i_{1}=1}^{\infty}, \ldots,\left(x_{i_{n}}^{n}\right)_{i_{n}=1}^{\infty}\right)=\left(T\left(x_{i_{1}}^{1}, \ldots, x_{i_{n}}^{n}\right)\right)_{i_{1}, \ldots, i_{n}=1}^{\infty}$ is continuous and verifies $\|\hat{T}\|=\pi_{\left(p ; q_{1}, \ldots, q_{n}\right)}(T)$.

As in the linear case, we have a composition theorem, which shows the good behavior of this class in relation to the $p$-summing linear operators. This result, from which we obtain a sharp result in the last section, is also applied in one of the main results in [12].

Theorem 2.3. Let $u_{j} \in \Pi_{q}\left(X_{j}, Y_{j}\right)$ and $T \in \Pi_{p}^{n}\left(Y_{1}, \ldots, Y_{n} ; Z\right)$ and let $1 \leq r<+\infty$ be such that $\frac{1}{r}=\frac{1}{p}+\frac{1}{q}$. Then $S=T\left(u_{1}, \ldots, u_{n}\right)$ is multiple r-summing and $\pi_{r}(S) \leq \pi_{p}(T) \prod_{j=1}^{n} \pi_{q}\left(u_{j}\right)$

Proof. The proof follows along the lines of [5, 2.22]. We consider $\left(x_{i_{j}}^{j}\right)_{i_{j}=1}^{\infty} \in$ $B\left(\ell_{r}^{\omega}\left(X_{j}\right)\right)$. By [5, Lemma 2.23], there are sequences $\left(\sigma_{i_{j}}^{j}\right)_{i_{j}=1}^{\infty} \in B\left(\ell_{q}\right),\left(y_{i_{j}}^{j}\right)_{i_{j}=1}^{\infty} \in$ $\ell_{p}\left(Y_{j}\right)$ such that $u_{j}\left(x_{i_{j}}^{j}\right)=\sigma_{i_{j}}^{j} y_{i_{j}}^{j}$ and $\left\|\left(y_{i_{j}}^{j}\right)_{i_{j}=1}^{\infty}\right\|_{p}^{\omega} \leq \pi_{q}\left(u_{j}\right)$.

As

$$
\left(\sum_{i_{1}, \ldots, i_{n}=1}^{\infty}\left\|T\left(y_{i_{1}}^{1}, \ldots, y_{i_{n}}^{n}\right)\right\|^{p}\right)^{\frac{1}{p}} \leq \pi_{p}(T) \prod_{j=1}^{n} \pi_{q}\left(u_{j}\right)
$$

and $\frac{r}{p}+\frac{r}{q}=1$, a straightforward use of Hölder's inequality gives

$$
\left(\sum_{i_{1}, \ldots, i_{n}=1}^{\infty}\left\|S\left(x_{i_{1}}^{1}, \ldots, x_{i_{n}}^{n}\right)\right\|^{r}\right)^{\frac{1}{r}} \leq \pi_{p}(T) \prod_{j=1}^{n} \pi_{q}\left(u_{j}\right)
$$

To finish this section we are going to state our main tool in the subsequent study of this class of operators. The proof follows immediately from the definitions.

Proposition 2.4. Let $T: X_{1} \times \cdots \times X_{n} \longrightarrow Y$ be a multilinear operator and let $1 \leq q_{1}, \ldots, q_{n} \leq p<\infty$. The following are equivalent

i) $T$ is multiple $\left(p ; q_{1}, \ldots, q_{n}\right)$-summing

ii) There exists a constant $K>0$ such that for every $m_{2}, \ldots, m_{n} \in \mathbb{N}$ and every choice of sequences $\left(x_{i_{j}}^{j}\right)_{i_{j}=1}^{m_{j}} \subset X_{j}$, with $\left\|\left(x_{i_{j}}^{j}\right)_{i_{j}=1}^{m_{j}}\right\|_{q_{j}}^{\omega} \leq 1(2 \leq j \leq n)$, we have that the associated linear operator

$$
S: X_{1} \longrightarrow \ell_{p}^{m_{2} \cdots m_{n}}(Y)
$$

given by

$$
S\left(x_{1}\right)=\left(T\left(x_{1}, x_{i_{2}}^{2}, \ldots, x_{i_{n}}^{n}\right)\right)_{i_{2}, \ldots, i_{n}=1}^{m_{2}, \ldots, m_{n}}
$$

is $\left(p, q_{1}\right)$-summing and verifies

$$
\pi_{\left(p, q_{1}\right)}(S) \leq K
$$

In that case, $\pi_{\left(p ; q_{1}, \ldots, q_{n}\right)}(T)=\min \{K: K$ verifies (2) $\}$ 


\section{Multiple summing multilinear operators on $\mathcal{L}_{\infty}$ SpaCes}

We state now a Grothendieck type theorem for multiple summing multilinear operators.

Theorem 3.1. Let $X_{j}$ be a $\mathcal{L}_{\infty, \lambda_{j}}$-space for $1 \leq j \leq n$ and let $Y$ be a space with cotype 2. Then, every multilinear operator $T: X_{1} \times \cdots \times X_{n} \longrightarrow Y$ is multiple 2-summing and

$$
\pi_{2}(T) \leq K_{n} \prod_{j=1}^{n} \lambda_{j}\|T\|
$$

where $K_{n}=\left(B_{4} C_{2}(Y)\right)^{2 n}$, with $C_{2}(Y)$ the cotype 2 constant of $Y$ and $B_{4}$ the constant of Khintchin's inequality [5, page 10].

Proof. We are going to proceed by induction. For $n=1$, the result is known (see [5, Theorem 11.14]).

Let us suppose then $n \geq 2$ and that the result is true for $n-1$. We are going to use the previous proposition. We consider $m_{2}, \ldots, m_{n} \in \mathbb{N}$ and sequences $\left(x_{i_{j}}^{j}\right)_{i_{j}=1}^{m_{j}} \subset$ $X_{j}$, with $\left\|\left(x_{i_{j}}^{j}\right)_{i_{j}=1}^{m_{j}}\right\|_{2}^{\omega} \leq 1(2 \leq j \leq n)$. We want to see that the associated linear operator

$$
S: X_{1} \longrightarrow \ell_{2}^{m_{2} \cdots m_{n}}(Y)
$$

given by

$$
S\left(x_{1}\right)=\left(T\left(x_{1}, x_{i_{2}}^{2}, \ldots, x_{i_{n}}^{n}\right)\right)_{i_{2}, \ldots, i_{n}=1}^{m_{2}, \ldots, m_{n}}
$$

verifies that $\pi_{2}(S) \leq K_{n}$.

By the $n-1$ case, we have that, for all $x_{1} \in X_{1}$, the operator $T_{x_{1}}=T\left(x_{1}, \cdot, \ldots, \cdot\right)$ : $X_{2} \times \cdots \times X_{n} \longrightarrow Y$ is multiple 2-summing and

$$
\pi_{2}\left(T_{x_{1}}\right) \leq K_{n-1} \prod_{j=2}^{n} \lambda_{j}\left\|T_{x_{1}}\right\| \leq K_{n-1} \prod_{j=2}^{n} \lambda_{j}\|T\|\left\|x_{1}\right\|
$$

So, for every $x_{1} \in X_{1}$,

$$
\left\|S\left(x_{1}\right)\right\| \leq \pi_{2}\left(T_{x_{1}}\right) \leq K_{n-1} \prod_{j=2}^{n} \lambda_{j}\|T\|\left\|x_{1}\right\|
$$

and then

$$
\|S\| \leq K_{n-1} \prod_{j=2}^{n} \lambda_{j}\|T\|
$$

Now, using [5, Theorem 11.12], we now that $\ell_{2}^{m_{2} \cdots m_{n}}(Y)$ has cotype 2 with constant $C_{2}\left(\ell_{2}^{m_{2} \cdots m_{n}}(Y)\right)=C_{2}(Y)$. Then, from the $n=1$ case, we obtain that $S$ is 2 summing and

$$
\pi_{2}(S) \leq K_{1} K_{n-1} \prod_{j=1}^{n} \lambda_{j}\|T\|=K_{n} \prod_{j=1}^{n} \lambda_{j}\|T\|
$$


See [12, Remark 3.9], where we solve a question stated in [17], for a direct application of Theorem 3.1.

Following the same reasoning we can prove the following improvement of $[4$, Theorem 6]

Theorem 3.2. Let $X_{j}$ be a Banach space for $1 \leq j \leq n$ and let $Y$ be a cotype $q$ space. Then, every multilinear operator $T: X_{1} \times \cdots \times X_{n} \longrightarrow Y$ is multiple $(q, 1)$-summing and

$$
\pi_{(q, 1)}(T) \leq C_{q}(Y)^{n}\|T\|
$$

where $C_{q}(Y)$ is the cotype $q$ constant of $Y$.

The proof is quite similar to the proof of Theorem 3.1, the only difference is to use in the induction step the following

Lemma 3.3. Let $X, Y$ be Banach spaces, where $Y$ is a cotype $q$ space, and let $(\Omega, \Sigma, \mu)$ be a measure space. Then, every linear operator $T: X \longrightarrow L_{q}(\mu, Y)$ is $(q, 1)$-summing and verifies that

$$
\pi_{(q, 1)}(T) \leq C_{q}(Y)\|T\|
$$

Proof. Let $\left(x_{i}\right)_{i=1}^{m} \subset X$. Since the formal inclusion $L_{q}[0,1] \hookrightarrow L_{2}[0,1]$ has norm 1 , we have that

$$
\begin{aligned}
\sum_{i=1}^{m}\left\|T\left(x_{i}\right)\right\|^{q} & =\sum_{i=1}^{m} \int_{\Omega}\left\|T\left(x_{i}\right)(\omega)\right\|_{Y}^{q} d \mu \leq C_{q}(Y)^{q} \int_{\Omega} \int_{0}^{1}\left\|T\left(\sum_{i=1}^{m} r_{i}(t) x_{i}\right)(\omega)\right\|^{q} d t d \mu \\
& \leq C_{q}(Y)^{q}\|T\|^{q} \int_{0}^{1}\left\|\sum_{i=1}^{m} r_{i}(t) x_{i}\right\|^{q} d t \leq C_{q}^{q}(Y)\|T\|^{q}\left(\left\|\left(x_{i}\right)_{i=1}^{m}\right\|_{1}^{\omega}\right)^{q}
\end{aligned}
$$

As an immediate consequence, we obtain the announced great improvement of $[4$, Corollary 7]

Corollary 3.4. If $q \geq 2$, every multilinear operator $T: c_{0} \times \cdots \times c_{0} \longrightarrow \ell_{q}$, verifies

$$
\left(\sum_{i_{1}, \ldots, i_{n} \geq 1}\left\|T\left(e_{i_{1}}, \ldots, e_{i_{n}}\right)\right\|^{q}\right)^{\frac{1}{q}} \leq\|T\|
$$

This corollary can be seen as a vector valued generalization of Littlewood's inequality (see [4] and the references therein to see this theorem and the history and importance of this kind of results):

Theorem 3.5 (Littlewood-Davie-Kaijser). If $T$ is a continuous $n$-linear form on $c_{0}$, then

$$
\left(\sum_{i_{1}, \ldots, i_{n}=1}^{\infty}\left|T\left(e_{i_{1}}^{1}, \ldots, e_{i_{n}}^{n}\right)\right|^{\frac{2 n}{n+1}}\right)^{\frac{n+1}{2 n}} \leq 2^{\frac{n-1}{2}}\|T\|
$$


In fact, using the Littlewood-Davie-Kaijser inequality, it is not difficult to obtain that every $n$-linear form on the product of Banach spaces is multiple $\left(\frac{2 n}{n+1} ; 1\right)$ summing (see [12] for details).

\section{Banach Lattices and multilinear Grothendieck's inequality}

We can improve our Theorem 3.1 if we consider the spaces to be Banach lattices. To this end, we consider, for $n \in \mathbb{N}$ and for $L$ a Banach lattice, the vector space $\widehat{\ell_{p}^{n}(L)}=\left\{\left(x_{1}, \ldots, x_{n}\right): x_{i} \in L\right\}$, with the norm given by

$$
\left\|\left(x_{i}\right)_{i=1}^{n}\right\|=\left\|\left(\sum_{i=1}^{n}\left|x_{i}\right|^{p}\right)^{\frac{1}{p}}\right\|
$$

and with the order given by

$$
\left(x_{i}\right)_{i=1}^{n} \leq\left(y_{i}\right)_{i=1}^{n} \Leftrightarrow x_{i} \leq y_{i} \text { for all } i
$$

It is easy to see that $\widetilde{\ell_{p}^{n}(L)}$ is a Banach lattice.

For our purposes it is enough to know (see [9]) that

$$
\left(\sum_{i=1}^{n}\left|x_{i}\right|^{p}\right)^{\frac{1}{p}}=\sup \left\{\sum_{i=1}^{m} a_{i} x_{i}: a_{i} \in B_{\ell_{p^{\prime}}^{m}}\right\}
$$

where the supremum is in the lattice sense (this supremum exists for every lattice) and $\frac{1}{p}+\frac{1}{p^{\prime}}=1$. Of course, when $L$ is some $C(K)$ or $L_{p}(\mu)$, it is easy to see that $\left(\sum_{i=1}^{n}\left|x_{i}\right|^{p}\right)^{\frac{1}{p}}$ coincide with the alternative pointwise definition.

Following [5, page 327], if $L$ is a Banach lattice and $x \in L$ we will note the ideal generated by $x$ as $I(x)$. We know that $I(x)$, with a suitable norm and the order inherited from $L$, can be identified (as a Banach lattice) with a $C(K)$ space.

The following simple lemma is probably known, but we have not been able to find a reference for it. We state its proof for completeness.

Lemma 4.1. Let $L$ be a Banach lattice, $1 \leq p<\infty$ and $n, m \in N$. We have that $\left.\widetilde{\ell_{p}^{m}} \widetilde{\left(\ell_{p}^{n}(L)\right.}\right)$ is identically isometric (as Banach lattice) to $\widetilde{\ell_{p}^{n m}(L)}$.

Proof. For $\left(x_{i j}\right)_{i, j=1}^{m, n} \subset L$, we set $x=\left(\sum_{i, j=1}^{m, n}\left|x_{i j}\right|^{p}\right)^{\frac{1}{p}} \in L$. So the norm of $\left(x_{i j}\right)_{i, j=1}^{m, n} \subset L$ in $\widetilde{\ell_{p}^{n m}(L)}$ is just $\|x\|_{L}$. Now, we know that the norm of $\left(x_{i j}\right)_{i, j=1}^{m, n} \subset L$ in $\ell_{p}^{m} \widetilde{\left(\ell_{p}^{n}(L)\right)}$ is

$$
\left\|\sup \left\{\sum_{i=1}^{m} a_{i} x_{i}: a_{i} \in B_{\ell_{p^{\prime}}^{m}}\right\}\right\|_{\widetilde{\ell_{p}^{n}(L)}}
$$

where $x_{i}=\left(x_{i j}\right)_{j=1}^{n} \in \widetilde{\ell_{p}^{n}(L)}$. By the definition of $\widetilde{\ell_{p}^{n}(L)}$, it is easy to see that 


$$
\begin{aligned}
\sup \left\{\sum_{i=1}^{m} a_{i} x_{i}: a_{i} \in B_{\ell_{p^{\prime}}^{m}}\right\} & =\left(\sup \left\{\sum_{i=1}^{m} a_{i} x_{i j}: a_{i} \in B_{\ell_{p^{\prime}}^{m}}\right\}\right)_{j=1}^{n} \\
& =\left(\left(\sum_{i=1}^{m}\left|x_{i j}\right|^{p}\right)^{\frac{1}{p}}\right)_{j=1}^{n} \in \widetilde{\ell_{p}^{n}(L)}
\end{aligned}
$$

Then, if $z=\left(\sum_{j=1}^{n}\left|\left(\sum_{i=1}^{m}\left|x_{i j}\right|^{p}\right)^{\frac{1}{p}}\right|^{p}\right)^{\frac{1}{p}}$, we have that the norm of $\left(x_{i j}\right)_{i, j=1}^{m, n}$ in $\widetilde{\ell_{p}^{m}} \widetilde{\left(\widehat{\ell_{p}^{n}(L)}\right)}$ is just $\|z\|_{L}$. As it is trivial that, in $I(x \vee z)$,

$$
\left(\sum_{j=1}^{n}\left|\left(\sum_{i=1}^{m}\left|x_{i j}\right|^{p}\right)^{\frac{1}{p}}\right|^{p}\right)^{\frac{1}{p}}=\left(\sum_{i, j=1}^{m, n}\left|x_{i j}\right|^{p}\right)^{\frac{1}{p}}
$$

we can conclude that $x=z$ (i.e. that the above is also true in $L$ ) by the following simple

Claim: Let $(L,\|\cdot\|)$ be a Banach lattice, $x_{1}, \ldots, x_{n} \in L$ and $x=\left(\sum_{i=1}^{n}\left|x_{i}\right|^{p}\right)^{\frac{1}{p}} \in$ $L$. Let us suppose that $I$ is an ideal of $L$ such that there exists a norm $\||\cdot|\|$ that makes $(S,|\|\cdot|\||)$ a Banach lattice with the inherited order. Then, if $x \in I$, we have that $x_{1}, \ldots, x_{n} \in I$ and that the element $\left(\sum_{i=1}^{n}\left|x_{i}\right|^{p}\right)^{\frac{1}{p}}$ in $I$ is just $x$.

In order to apply our results in the complex case too, we have to define the complexification of a Banach lattice. Following [9, page 43] or [18, II.11], given a Banach lattice $L$, we define its complexification $L^{\mathbb{C}}$ as $L \oplus L$ with the scalar product given by $(a+i b)(x, y)=(a x-b y, a y+b x)$ and the norm given by $\|(x, y)\|=$ $\||(x, y)|\|_{L}$, where the absolute value is defined as $|(x, y)|=\left(|x|^{2}+|y|^{2}\right)^{\frac{1}{2}} \in L$. Given $z=(x, y) \in L^{\mathbb{C}}$, we will note $\operatorname{Re}(z)=x$ and $\operatorname{Im}(z)=y$.

As expected, the complexification of $L_{p}(\mu)$ or $C(K)$ are the corresponding complex spaces.

The main theorem of this section is the following generalization of the Krivine's version of Grothendieck's theorem.

Theorem 4.2. Let $L_{j}, L$ be Banach lattices.

(1) Let $\left(x_{i_{j}}^{j}\right)_{i_{j}=1}^{m_{j}} \subset L_{j}$. Then, every multilinear operator $T: L_{1} \times \cdots \times L_{n} \longrightarrow L$ verifies that

$$
\left\|\left(\sum_{i_{1}, \ldots, i_{n}=1}^{m_{1}, \ldots, m_{n}}\left|T\left(x_{i_{1}}^{1}, \ldots, x_{i_{n}}^{n}\right)\right|^{2}\right)^{\frac{1}{2}}\right\| \leq K_{G, \mathbb{R}}^{n}\|T\| \prod_{j=1}^{n}\left\|\left(\sum_{i_{j}=1}^{m_{j}}\left|x_{i_{j}}^{j}\right|^{2}\right)^{\frac{1}{2}}\right\|
$$

(2) Let $\left(z_{i_{j}}^{j}\right)_{i_{j}=1}^{m_{j}} \subset L_{j}^{\mathbb{C}}$. Then, every multilinear operator $T: L_{1}^{\mathbb{C}} \times \cdots \times L_{n}^{\mathbb{C}} \longrightarrow$ $L^{\mathbb{C}}$ verifies that 


$$
\left\|\left(\sum_{i_{1}, \ldots, i_{n}=1}^{m_{1}, \ldots, m_{n}}\left|T\left(z_{i_{1}}^{1}, \ldots, z_{i_{n}}^{n}\right)\right|^{2}\right)^{\frac{1}{2}}\right\| \leq K_{G, \mathbb{C}}^{n}\|T\| \prod_{j=1}^{n}\left\|\left(\sum_{i_{j}=1}^{m_{j}}\left|z_{i_{j}}^{j}\right|^{2}\right)^{\frac{1}{2}}\right\|
$$

Proof. The real case is an immediate consequence of Krivine's theorem (see [9, Theorem 1.f.14]), Lemma 4.1 and the induction techniques used in Theorem 3.1 or in the complex case below. Let us prove now the complex case.

Following the reasonings of [9, Theorem 1.f.14], and using Lemma 4.1 when needed, it is not difficult to prove the case $n=1$. We suppose then $n \geq 2$ and the result to be true for $n-1$. Let us consider Banach lattices $L_{1}, \ldots, L_{n}, L$ and a multilineal operator $T: L_{1}^{\mathbb{C}} \times \cdots \times L_{n}^{\mathbb{C}} \longrightarrow L^{\mathbb{C}}$. Let us fix $\left(z_{i_{j}}^{j}\right)_{i_{j}=1}^{m_{j}} \subset L_{j}$ such that

$$
\left\|\left(\sum_{i_{j}=1}^{m_{j}}\left|z_{i_{j}}^{j}\right|^{2}\right)^{\frac{1}{2}}\right\|=1
$$

By the linear case, the linear operator $S: L_{1}^{\mathbb{C}} \longrightarrow\left(\ell_{2}^{m_{2} \cdots m_{n}}(L)\right)^{\mathbb{C}}$ given by

$$
S\left(z^{1}\right)=\left(\left(\operatorname{Re} T\left(z^{1}, z_{i_{2}}^{2}, \ldots, z_{i_{n}}^{n}\right)\right)_{i_{2}, \ldots, i_{n}=1}^{m_{2}, \ldots, m_{n}},\left(\operatorname{Im} T\left(z^{1}, z_{i_{2}}^{2}, \ldots, z_{i_{n}}^{n}\right)\right)_{i_{2}, \ldots, i_{n}=1}^{m_{2}, \ldots, m_{n}}\right)
$$

satisfies

$$
\left\|\left(\sum_{i_{1}=1}^{m_{1}}\left|S\left(z_{i_{1}}^{1}\right)\right|^{2}\right)^{\frac{1}{2}}\right\| \leq K_{G, \mathbb{C}}\|S\|
$$

By Lemma 4.1 and the arguments therein, we have that

$$
\left\|\left(\sum_{i_{1}=1}^{m_{1}}\left|S\left(z_{i_{1}}^{1}\right)\right|^{2}\right)^{\frac{1}{2}}\right\|=\left\|\left(\sum_{i_{1}, \ldots, i_{n}=1}^{m_{1}, \ldots, m_{n}}\left|T\left(z_{i_{1}}^{1}, \ldots, z_{i_{n}}^{n}\right)\right|^{2}\right)^{\frac{1}{2}}\right\|
$$

and that

$$
\left\|S\left(z^{1}\right)\right\|=\left\|\left(\left|\operatorname{Re} S\left(z^{1}\right)\right|^{2}+\left|\operatorname{Im} S\left(z^{1}\right)\right|^{2}\right)^{\frac{1}{2}}\right\|=\left\|\left(\left|T\left(z^{1}, z_{i_{2}}^{2}, \ldots, z_{i_{n}}^{n}\right)\right|\right)_{i_{2}, \ldots, i_{n}=1}^{m_{1}, \ldots, m_{n}}\right\|
$$

To conclude, we can use (3) and the $n-1$ case to prove that $\|S\| \leq K_{G, \mathbb{C}}^{n-1}$.

We can obtain immediately an improvement of the constant in Theorem 3.1 for the most important case.

Corollary 4.3. Let $X_{j}$ be a $\mathcal{L}_{\infty, \lambda_{j}}$ space for $1 \leq j \leq n$ and $Y$ a $\mathcal{L}_{1, \lambda}$ space. Then, every multilinear operator $T: X_{1} \times \cdots \times X_{n} \longrightarrow Y$ is multiple 2-summing and it verifies

$$
\pi_{2}(T) \leq K_{G}^{n} \prod_{j=1}^{n} \lambda_{j}\|T\|
$$


Proof. By a localization argument, all we have to do is to prove (4) in the case $X_{j}=\ell_{\infty}^{k_{j}}$ and $Y=\ell_{1}^{k}$. But this is trivial, using Theorem 4.2 and the fact that

$$
\begin{gathered}
\left\|\left(\sum_{i_{j}=1}^{m_{j}}\left|x_{i_{j}}^{j}\right|^{2}\right)^{\frac{1}{2}}\right\|=\left\|\left(x_{i_{j}}^{j}\right)_{i_{j}=1}^{m_{j}}\right\|_{2}^{\omega} \quad \text { for all }\left(x_{i_{j}}^{j}\right)_{i_{j}=1}^{m_{j}} \subset \ell_{\infty}^{k_{j}} \\
\left\|\left(\sum_{i=1}^{m}\left|y_{i}\right|^{2}\right)^{\frac{1}{2}}\right\| \geq\left(\sum_{i=1}^{m}\left\|y_{i}\right\|^{2}\right)^{\frac{1}{2}} \quad \text { for all }\left(y_{i}\right)_{i=1}^{m} \subset \ell_{1}^{k}
\end{gathered}
$$

We can now give an alternative proof of one of the known multilinear extensions of Grothendieck's inequality (see for example [19, Inequality C(r)]). Our proof is quite simple, it covers both the real and complex case and it shows clearly the optimality of the constant.

Corollary 4.4. Let $n \geq 2$ and $\left(a_{i_{1}, \ldots, i_{n}}\right)_{i_{1}, \ldots, i_{n}=1}^{m_{1}, \ldots, m_{n}} \subset \mathbb{K}$. Let us consider sequences $\left(x_{i_{1}}^{1}\right)_{i_{1}=1}^{m_{1}} \subset B\left(\ell_{2}^{k_{1}}\right), \ldots,\left(x_{i_{n-1}}^{n-1}\right)_{i_{n-1}=1}^{m_{n-1}} \subset B\left(\ell_{2}^{k_{n-1}}\right)$ and $\left(b_{i_{n}}\right)_{i_{n}=1}^{m_{n}} \subset B\left(\ell_{2}^{k_{1} \cdots k_{n-1}}\right)$. Then the following holds

$$
\begin{aligned}
& \left|\sum_{i_{1}, \ldots, i_{n}=1}^{m_{1}, \ldots, m_{n}} a_{i_{1}, \ldots, i_{n}} \sum_{r_{1}, \ldots, r_{n-1}=1}^{k_{1}, \ldots, k_{n-1}} x_{i_{1}}^{1}\left(r_{1}\right) \cdots x_{i_{n-1}}^{n-1}\left(r_{n-1}\right) b_{i_{n}}\left(r_{1}, \ldots, r_{n-1}\right)\right| \leq \\
& K_{G}^{n-1} \sup \left\{\left|\sum_{i_{1}, \ldots, i_{n}=1}^{m_{1}, \ldots, m_{n}} a_{i_{1}, \ldots, i_{n}} t_{i_{1}} \cdots t_{i_{n}}\right|:\left|t_{i_{1}}\right| \leq 1, \ldots,\left|t_{i_{n}}\right| \leq 1\right\}
\end{aligned}
$$

Moreover, the constant $K_{G}^{n-1}$ is optimal.

Proof. Let us consider $T: \ell_{\infty}^{m_{1}} \times \cdots \times \ell_{\infty}^{m_{n-1}} \longrightarrow \ell_{1}^{m_{n}}$ given by $T\left(e_{i_{1}}^{1}, \ldots, e_{i_{n-1}}^{n-1}\right)=$ $\sum_{i_{n}=1}^{m_{n}} a_{i_{1}, \ldots, i_{n}} e_{i_{n}}^{n}$. We know that

$$
\|T\|=\sup \left\{\left|\sum_{i_{1}, \ldots, i_{n}=1}^{m_{1}, \ldots, m_{n}} a_{i_{1}, \ldots, i_{n}} t_{i_{1}} \cdots, t_{i_{n}}\right|:\left|t_{i_{1}}\right| \leq 1, \ldots,\left|t_{i_{n}}\right| \leq 1\right\}
$$

For every $j \in\{1, \ldots, n-1\}$ we consider the sequence $\left(y_{r_{j}}^{j}\right)_{r_{j}=1}^{k_{j}} \subset \ell_{\infty}^{m_{j}}$ defined by $y_{r_{j}}^{j}\left(i_{j}\right)=x_{i_{j}}^{j}\left(r_{j}\right)$. Since $\left(x_{i_{j}}^{j}\right)_{i_{j}=1}^{m_{j}} \subset B\left(\ell_{2}^{k_{j}}\right)$, we have that

$$
\left\|\left(\sum_{r_{j}=1}^{k_{j}}\left|y_{r_{j}}^{j}\right|^{2}\right)^{\frac{1}{2}}\right\| \leq 1
$$

and Theorem 4.2 tells us that

$$
\left\|\left(\sum_{r_{1}, \ldots, r_{n-1}=1}^{k_{1}, \ldots, k_{n-1}}\left|T\left(y_{r_{1}}^{1}, \ldots, y_{r_{n-1}}^{n-1}\right)\right|^{2}\right)^{\frac{1}{2}}\right\| \leq K_{G}^{n-1}
$$


Now, it is not hard to see that

$$
\begin{gathered}
\left\|\left(\sum_{r_{1}, \ldots, r_{n-1}=1}^{k_{1}, \ldots, k_{n-1}}\left|T\left(y_{r_{1}}^{1}, \ldots, y_{r_{n-1}}^{n-1}\right)\right|^{2}\right)^{\frac{1}{2}}\right\|= \\
=\sup \left\{\sum_{i_{n}=1}^{m_{n}} \sum_{r_{1}, \ldots, r_{n-1}=1}^{k_{1}, \ldots, k_{n-1}} c_{i_{n}}\left(r_{1}, \ldots, r_{n-1}\right) \sum_{i_{1}, \ldots, i_{n-1}=1}^{m_{1}, \ldots, m_{n-1}} a_{i_{1}, \ldots, i_{n}} y_{r_{1}}^{1}\left(i_{1}\right) \cdots y_{r_{n-1}}^{n-1}\left(i_{n-1}\right)\right\}
\end{gathered}
$$

where the supremum is taken over all the sequences $c_{i_{n}} \in B\left(\ell_{2}^{k_{1} \cdots k_{n-1}}\right)$, so we are done.

To see the optimality of the constant, we note that the argument above can be reversed. So it is enough to find, for each $\epsilon>0$, natural numbers $m_{j}, k_{j}$, sequences $\left(y_{r_{j}}^{j}\right)_{r_{j}=1}^{k_{j}} \subset \ell_{\infty}^{m_{j}}$ with $\left\|\left(y_{r_{j}}^{j}\right)_{r_{j}=1}^{k_{j}}\right\|_{2}^{\omega} \leq 1$, and a multilinear operator $T$ : $\ell_{\infty}^{m_{1}} \times \cdots \times \ell_{\infty}^{m_{n-1}} \longrightarrow \ell_{1}^{m_{n}}$ with $\|T\|=1$ and

$$
\left\|\left(\sum_{r_{1}, \ldots, r_{n-1}=1}^{k_{1}, \ldots, k_{n-1}}\left|T\left(y_{r_{1}}^{1}, \ldots, y_{r_{n-1}}^{n-1}\right)\right|^{2}\right)^{\frac{1}{2}}\right\| \geq\left(K_{G}-\epsilon\right)^{n-1}
$$

By the linear case (n=2 above) there exists a linear operator $R: \ell_{\infty}^{m} \longrightarrow \ell_{1}^{s}$ with $\|R\|=1$ and a sequence $\left(y_{r}\right)_{r=1}^{k} \subset \ell_{\infty}^{m}$ with $\left\|\left(y_{r}\right)_{r=1}^{k}\right\|_{2}^{\omega} \leq 1$ and such that $\left\|\left(\sum_{r=1}^{k}\left|R\left(y_{r}\right)\right|^{2}\right)^{\frac{1}{2}}\right\| \geq K_{G}-\epsilon$. To finish, we just need to consider $m_{j}=m, k_{j}=k$ for $1 \leq j \leq n-1, m_{n}=s^{n-1}, y_{r}^{j}=y_{r}$ and

$$
T=R \otimes \cdots \otimes R: \ell_{\infty}^{m} \times \cdots \times \ell_{\infty}^{m} \longrightarrow \ell_{1}^{s} \otimes_{\pi} \cdots \otimes_{\pi} \ell_{1}^{s}=\ell_{1}^{s^{n-1}}
$$

\section{Multiple Summing OPERAtors on $\mathcal{L}_{1}$ SPACES}

In this section we extend a famous result of [8] stating that every linear operator from $L_{1}(\mu)$ to a Hilbert space is 1-summing, with $\pi_{1}(T) \leq K_{G}\|T\|$ (see, for instance, $[5$, Theorem 3.1] or [16, Theorem 5.12]).

We need first

Theorem 5.1. Let $X_{j}$ be a $\mathcal{L}_{1, \lambda_{j}}$-space $(1 \leq j \leq n)$ and let $H$ be a Hilbert space. Then, every multilinear operator $T: X_{1} \times \cdots \times X_{n} \longrightarrow H$ is multiple 2-summing and

$$
\pi_{2}(T) \leq \tilde{K}_{G}^{n} \prod_{j=1}^{n} \lambda_{j}\|T\|
$$

where $\tilde{K}_{G}$ is $\left(\frac{\pi}{2}\right)^{\frac{1}{2}}$ in the real case and $\frac{2}{\sqrt{\pi}}$ in the complex case.

Proof. The proof is again an application of Proposition 2.4, using the same inductive techniques as in the proof of Theorem 3.1 and using [16, Theorem 5.10] for the case $n=1$.

Now we can prove 
Theorem 5.2. Let $X_{j}$ be a $\mathcal{L}_{1, \lambda_{j}}$-space $(1 \leq j \leq n)$ and let $H$ be a Hilbert space. Then, every multilinear operator $T: X_{1} \times \cdots \times X_{n} \longrightarrow H$ is multiple 1-summing and

$$
\pi_{1}(T) \leq\left(\tilde{K}_{G} K_{G}\right)^{n} \prod_{j=1}^{n} \lambda_{j}\|T\|
$$

Proof. By a standard localization argument, we can suppose that $X_{j}=\ell_{1}^{k_{j}}$. Let $T: \ell_{1}^{k_{1}} \times \cdots \times \ell_{1}^{k_{n}} \longrightarrow H$ be a multilinear operator and let us choose $m_{j} \in \mathbb{N}$ and sequences $\left(x_{i_{j}}^{j}\right)_{i_{j}=1}^{m_{j}} \subset \ell_{1}^{k_{j}}$, with $\left\|\left(x_{i_{j}}^{j}\right)_{i_{j}=1}^{m_{j}}\right\|_{1}^{\omega} \leq 1(1 \leq j \leq n)$.

For each $j \in\{1, \ldots, n\}$, let us consider the operator $u_{j}: \ell_{\infty}^{m_{j}} \longrightarrow \ell_{1}^{k_{j}}$ defined by $u_{j}\left(e_{i_{j}}\right)=x_{i_{j}}^{j}$. Then $\left\|u_{j}\right\|=\left\|\left(x_{i_{j}}^{j}\right)_{i_{j}=1}^{m_{j}}\right\|_{1}^{\omega} \leq 1$. Therefore, by [5, Lemma 3.6], $\pi_{2}\left(u_{j}\right) \leq K_{G}$.

We consider now the operator $S=T\left(u_{1}, \ldots, u_{j}\right): \ell_{\infty}^{m_{1}} \times \cdots \times \ell_{\infty}^{m_{n}} \longrightarrow H$

According to Theorem 5.1, we have that

$$
\pi_{2}(T) \leq \tilde{K}_{G}^{n}\|T\|
$$

So, Theorem 2.3 tells us that

$$
\pi_{1}(S) \leq\left(\tilde{K}_{G} K_{G}\right)^{n}\|T\|
$$

Therefore, using that $\left\|\left(e_{i_{j}}\right)_{i_{j}=1}^{m_{j}}\right\|_{1}^{\omega}=1$, we conclude

$$
\begin{aligned}
& \sum_{i_{1}, \ldots, i_{n}=1}^{m_{1}, \ldots, m_{n}}\left\|T\left(x_{i_{1}}^{1}, \ldots, x_{i_{n}}^{n}\right)\right\|=\sum_{i_{1}, \ldots, i_{n}=1}^{m_{1}, \ldots, m_{n}}\left\|S\left(e_{i_{1}}, \ldots, e_{i_{n}}\right)\right\| \leq \\
& \leq \pi_{1}(S)\left\|\left(e_{i_{1}}\right)_{i_{1}=1}^{m_{1}}\right\|_{1}^{\omega} \ldots\left\|\left(e_{i_{n}}\right)_{i_{n}=1}^{m_{n}}\right\|_{1}^{\omega} \leq\left(\tilde{K}_{G} K_{G}\right)^{n}\|T\|,
\end{aligned}
$$

Remark 5.3. We have deduced Theorem 5.2 from Theorem 5.1 and Theorem 2.3. Note that, since we have not yet been able to prove an inclusion theorem, Theorem 5.1 does not follow, at least not trivially, from Theorem 5.2. Note also that the linear version of Theorem 5.1 corresponds to the "dual" version of the "little Grothendieck's theorem" (see [16]). Finally, we want to remark that the constant we get in Theorem 5.2 is far from optimal when $n=1$. We think the constant should be $K_{G}^{n} \prod_{j=1}^{n} \lambda_{j}$, but we have not been able to prove this.

Remark 5.4. In the results above, we can change (the constants will also change) each $\mathcal{L}_{1}$-space by any G.T. space with cotype 2 (see [16, Chapter 6] for definition and references). Note that, to our best knowledge, there are not known examples of G.T. spaces without cotype 2.

As mentioned before, there are several generalizations of Grothendieck's matrix inequality ([3], [19]). Next we state one more such generalization, apparently new. Standard techniques show that this inequality is equivalent to Theorem 5.2. 
Theorem 5.5. Let $n \geq 2$. For every $l \in\{1, \ldots n\}$, let $\left(a_{i_{l}, j_{l}}\right)_{i_{l}, j_{l}=1}^{p_{l}, q_{l}} \subset \mathbb{K}$ be a matrix such that

$$
\sup \left\{\left|\sum_{i_{l}, j_{l}=1}^{p_{l}, q_{l}} a_{i_{l}, j_{l}} t_{i_{l}} t_{j_{l}}^{\prime}\right|:\left|t_{i_{l}}\right| \leq 1,\left|t_{j_{l}}^{\prime}\right| \leq 1\right\} \leq 1
$$

For any given Hilbert space $H$, consider sequences $\left(x_{i_{1}, \ldots, i_{n}}\right)_{i_{1}, \ldots, i_{n}=1}^{p_{1}, \ldots, p_{n}} \subset B_{H}$, $\left(y_{j_{1}, \ldots, j_{n}}\right)_{j_{1}, \ldots, j_{n}=1}^{q_{1}, \ldots, q_{n}} \subset B_{H}$. Then

$$
\left|\sum_{i_{1}, \ldots, i_{n}, j_{1} \ldots, j_{n}=1}^{p_{1}, \ldots, p_{n}, q_{1} \ldots, q_{n}} a_{i_{1}, j_{1}} \cdots a_{i_{n}, j_{n}}\left\langle x_{i_{1}, \ldots, i_{n}}, y_{j_{1}, \ldots, j_{n}}\right\rangle\right| \leq\left(\tilde{K}_{G} K_{G}\right)^{n}
$$

To finish, let us mention that Theorem 5.2 is also equivalent to saying that, for any measures $\mu_{1}, \ldots, \mu_{n}$, if the sequences $\left(x_{i_{l}}^{l}\right)_{i_{l}} \subset L_{1}\left(\mu_{l}\right)(1 \leq l \leq n)$ are weakly summable, then the sequence $\left(x_{i_{1}}^{1} \otimes \cdots \otimes x_{i_{n}}^{n}\right)_{i_{1} \ldots, i_{n}} \subset L_{1}\left(\mu_{1}\right) \otimes_{\pi} \cdots \otimes_{\pi} L_{1}\left(\mu_{n}\right)=$ $L_{1}\left(\mu_{1} \times \cdots \times \mu_{n}\right)$ is also weakly summable. The interested reader can check that this, in turn, can also be read as an inequality concerning certain matrixes.

\section{REFERENCES}

1. R. Alencar and M.C. Matos, Some classes of multilinear mappings between Banach spaces, 1989, Publicaciones del Departamento de Análisis Matemático, sec. 1, no. 12. Universidad Complutense de Madrid.

2. R.C. Blei, Multilinear measure theory and the Grothendieck factorization theorem, Proceedings of the London Mathematical Society 56 (1988), no. 3, 529-546.

3. T.K. Carne, Banach lattices and extensions of Grothendieck's inequality, Journal of the London Mathematical Society 21 (1980), 496-516.

4. Y.S. Choi, S.G. Kim, Y. Meléndez, and A. Tonge, Estimates for absolutely summing norms of polynomials and multilinear maps, Quart. J. Math. 52 (2001), 1-12.

5. J. Diestel, H. Jarchow, and A. Tonge, Absolutely summing operators, Cambridge University Press, 1995.

6. V. Dimant, Strongly p-summing multilinear operators, Preprint.

7. A. Grothendieck, Résumé de la théorie métrique des produits tensoriels topologiques, Bol. Soc. Mat. Sao Paulo 8 (1956), 1-79.

8. J. Lindenstrauss and A. Pelczynski, Absolutely summing operators in $\mathcal{L}_{p}$-spaces and their applications, Studia Mathematica 29 (1968), 257-326.

9. J. Lindenstrauss and L. Tzafriri, Classical Banach spaces II, Springer-Verlag, 1977.

10. M.C. Matos, Absolutely summing holomorphic mappings, An. Acad. Bras. Ci. 68 (1996), 1-13.

11. D. Pérez-García, Operadores multilineales absolutamente sumantes, Dissertation. Universidad Complutense de Madrid, 2002.

12. D. Pérez-García and I. Villanueva, Multiple summing operators on $C(K)$ spaces, Preprint.

13. _ Multiple summing operators on Hilbert spaces, Preprint.

14. A. Pietsch, Absolut p-summierende Abbildungen in normierten Räumen, Studia Mathematica 28 (1967), 333-353.

15. _ Ideals of multilinear functionals (designs of a theory), Proceedings of the Second International Conference on Operator Algebras, Ideals, and their Applications in Theoretical Physics (Leipzig), Teubner-Texte, 1983, pp. 185-199.

16. G. Pisier, Factorization of linear operators and geometry of Banach spaces, CBMS Regional Conference Series, no. 60, American Mathematical Society, 1986.

17. M.S. Ramanujan and E. Schock, Operator ideals and spaces of bilinear operators, Linear and Multilinear Algebra 18 (1985), 307-318.

18. H.H. Schaefer, Banach lattices and positive operators, Springer-Verlag, 1974.

19. A. Tonge, The Von Neumann inequality for polynomials in several Hilbert-Schmidt operators, Journal of the London Mathematical Society 18 (1978), 519-526. 
20. I. Villanueva, Integral mappings between Banach spaces, to appear in J. Math. Anal. Appl.

E-mail address: bombal@mat.ucm.es, dperez@mat.ucm.es, ignaciov@mat.ucm.es

Departamento de Análisis Matemático, Facultad de Matemáticas, Universidad ComPLutense De MAdrid, MAdRID 28040 